\title{
3GPP NB-IoT, tecnología y herramientas de medida
}

\author{
Almudena Díaz Zayas, Pedro Merino Gómez \\ Departamento de Lenguajes y Ciencias de la Computación, \\ Universidad de Málaga, Andalucía Tech, \\ Edificio de Investigación Ada Byron, Málaga, España, 29071 \\ almudiaz@1cc.uma.es,pedro@1cc.uma.es
}

\author{
F. Javier Rivas Tocado \\ Keysight Technologies \\ Málaga, Spain \\ Email: javi_rivas@keysight.com
}

\begin{abstract}
Resumen-La primera versión de los estándares 3GPP Narrow Band-IoT (NB-IoT) se finalizó en Junio de 2016 como parte de la Release 13. NB-IoT es una nueva tecnología de acceso radio que puede coexistir con los despliegues actuales de GSM, UMTS y LTE. De hecho, la especificación de NB-IoT se ha integrado en los estándares LTE. NBIoT va un paso más allá que la especificación de MTC (Machine Type Communication), enfocándose en dispositivos de extremadamente bajo coste, despliegues masivos y tasas de transmisión reducidas con un ancho de banda de solamente $200 \mathrm{kHz}$ (de ahí su nombre). En este artículo realizaremos una revisión en detalle de las especificaciones 3GPP destacando las modificaciones necesarias sobre los despliegues LTE tradicionales para proporcionar conectividad a dispositivos de usuario (UEs) Cat-NB1. Asimismo, introduciremos una perspectiva novedosa sobre las ventajas de usar de nuevas herramientas y soluciones para el análisis y medida de comunicaciones NB-IoT, como el UXM E7515A de Keysight Technologies, proporcionando también lecciones aprendidas en el incipiente uso de esta nueva tecnología.
\end{abstract}

Palabras Clave-LTE, Narrowband-IoT, Machine Type Communication, entorno de pruebas, Internet de las cosas

\section{INTRODUCCIÓN}

Como resultado del study item TR 45.820 [1] de 3GPP, titulado 'Cellular system support for ultra-low complexity and low throughput Internet of Things (CIoT)', se ha propuesto una nueva tecnología radio llamada NB-IoT. NB-IoT se está integrando en los estándares LTE y se ha estandarizado una nueva categoría de dispositivos (UEs) in [2], llamada Cat-NB1. Las funcionalidades proporcionadas por NB-IoT se han introducido previamente en [4][5] y están orientadas a cubrir el segmento inferior de las comunicaciones máquina a máquina, también conocidas como Machine Type Communication (MTC): cobertura extendida, soporte de un número masivo de dispositivos de baja tasa de transmisión, alta latencia permitida, dispositivos de ultra-bajo coste, bajo consumo de potencia y arquitectura de red optimizada.

Como se muestra en la Tabla I, los requerimientos de
NB-IoT son los mismos que para eMTC (enhanced MTC) [3], pero con el foco en escenarios masivos MTC en el segmento de bajo coste. NB-IoT proporciona un coste por dispositivo incluso inferior que eMTC, y una mayor extensión de cobertura con un presupuesto de pérdidas de propagación para el enlace (MCL) de $164 \mathrm{~dB}$ (al menos en despliegue aislado). La duración de la batería se ha buscado que pueda alcanzar los 10 años con una batería de 5 Vatios Hora. NB-IoT es compatible con los modos mejorados de recepción discontinua (eDRX) introducidos en la Release 13 de 3GPP para reducir el consumo de potencia. El número de dispositivos soportado por celda es de unos 55.000.

Al contrario que eMTC, que únicamente puede ser desplegada en modo in-band, utilizando bloques de recursos dentro de una portadora LTE normal, NB-IoT puede desplegarse también en los bloques de recursos sin usar dentro de la banda de guarda (guard-band) de la portadora LTE, o en modo aislado (standalone) para despliegues en espectro dedicado. NB-IoT resulta particularmente adecuado para el reaprovechamiento de los canales GSM.

Una prueba de concepto inicial se llevó a cabo por la Universidad de Aalto y Ericcson Research en Finlandia. Estos test iniciales tuvieron lugar en modo standalone y usando una arquitectura Cloud RAN (C-RAN). La capacidad de NB-IoT de soportar altas latencias lo hace adaptarse muy bien a los caos de uso que involucran despliegues basados en C-RAN.

En este artículo describimos la evolución de los estándares 3GPP relacionados con MTC/NB-IoT y detallamos los cambios introducidos en LTE para proporcionar soporte a NB-IoT, el último estándar de 3GPP para IoT. El artículo está organizado como sigue. En la sección II, proporcionamos el contexto necesario sobre los estándares 3GPP para MTC/IoT para entender el papel de NB-IoT en la era IoT. La sección III describe las modificaciones introducidas en la red de acceso radio. La transformación 


\begin{tabular}{|c|c|c|}
\hline & eMTC (Enhanced MTC) Release 13 & NB-IoT (Release 13) \\
\hline \hline Coverage Enhancement & 15 dB gain better compared to GPRS & 20 dB gain compared to GPRS \\
\hline Reduced complexity & Similar to EGPRS modem cost & Ultra-low cost $(\sim 1 \$)$ \\
\hline Power efficiency & 10 year battery life & 10 year battery life \\
\hline Latency & 10 sec & 10 sec \\
\hline Capacity & $\sim 10000$ devices per cell & $\sim 50000$ decives per cell \\
\hline Coexistence & LTE in-band & GSM standalone, LTE in-band, LTE guard-band \\
\hline \multicolumn{2}{|c}{ Cuadro I }
\end{tabular}

EMTC VS NB-IOT FEATURES

arquitectural sufrida en el EPC se explica en la sección IV. En la sección $\mathrm{V}$ realizamos una aproximación novedosa a NB-IoT desde un punto de vista de prueba y medida, adelantando unas primeras lecciones aprendidas. Finalmente, en la sección VI, proporcionamos una comparación adicional entre MTC y NB-IoT, e introducimos mejoras adicionales de Releases posteriores.

\section{CONTEXTO}

3GPP (Third Generation Partnership Project) es el organismo de estandarización que especifica los sistemas de comunicaciones móviles LTE/LTE-Advanced, así como 3G UTRA y 2G GSM. Los estándares 3GPP están estructurados en Releases. La definición de la tecnología móvil conocida como Long Term Evolution (LTE) se inició en 2005 y las primeras especificaciones 3GPP se introdujeron en la Release 8 en Diciembre de 2007. El término 'user equipment' (UE), se ha usado tradicionalmente por 3GPP para referirse a los dispositivos celulares utilizados por los suscriptores para acceder a los servicios de red móvil. Un UE puede ser un Smartphone o un dispositivo empotrado contenido en un equipo de comunicaciones máquina a máquina (M2M). Para soportar múltiples tipos de UE con distintas capacidades, 3GPP define categorías diferentes en 3GPP 36.306. Las categorías difieren en las máximas tasas de transmisión soportadas en los enlaces ascendente y descendente, que también están asociadas, por ejemplo, con el soporte de multiplexación espacial (transmisión MIMO). También se utilizan las categorías en los eNodeB (estacione base LTE) para determinar las condiciones bajo las cuales tendrá lugar la comunicación con el UE. Como se elaborará en más detalle a continuación, el concepto de categoría de UE tiene mucha importancia en IoT. La Tabla II proporciona un resumen de las categorías de terminales de usuario orientadas a escenarios MTC/IoT.

La versión inicial de los estándares LTE Machine Type Communication (MTC) se introdujo en la release 8 y estaba basada en la Categoría 1. La Categoría 1 era la de menor capacidad con una tasa de transmisión máxima de $10 \mathrm{Mbs}$ en el enlace descendente y de $5 \mathrm{Mbps}$ en el enlace ascendente. Por otra parte la Categoría 5, la de mayor capacidad en Release 8, soportaba tasas de transmisión de 300Mbps en el enlace descendente y $75 \mathrm{Mbps}$ en el ascendente. Aunque los dispositivos con Categoría 1 no soportan transmisión MIMO, aun así, incorporan 2 antenas receptoras y soportan todas las opciones de ancho de canal de RF desde 1.4 a $20 \mathrm{MHz}$. Además, esta categoría no cumple con los requerimientos de duración de batería, coste y rango necesarios para IoT. La primera Release de MTC estaba enfocada en la optimización de los mecanismos de tarificación, direccionamiento, ubicación fija, poca movilidad y baja actividad de los terminales, manejo de un gran volumen de suscripciones y de datos de usuario en la red, gestión de problemas en servicios M2M y en aspectos de seguridad

En la Release 12 se introdujo una nueva categoría de UE, la Categoría 0, que proporciona reducciones de coste de aproximadamente el $50 \%$ comparada con la Categoría 1. También se introdujo un nuevo modo de ahorro de potencia en esta Release, en 3GPP TS 24.301 y 23.682. El principal propósito de esta nueva funcionalidad es reducir el consumo de energía mientras que el dispositivo no está transmitiendo ni recibiendo. Otras soluciones para MTC se discutieron en Release 12 como parte de 3GPP 36.888. La especificación de MTC UE Cat0 incorpora otras mejoras como la transmisión half-duplex, una única cadena de RF y la reducción de la tasa pico de transmisión. La reducción de coste usando un ancho de banda reducido y las mejoras de cobertura se pospusieron a la Release 13.

Continuando el trabajo normativo iniciado en Release 12 para mejorar la adecuación de LTE al prometedor mercado IoT, el objetivo clave de eMTC (frecuentemente referenciado como LTE-M) en Release 13 es la definición de una nueva categoría de UE de baja complejidad que soporte un ancho de banda reducido, baja potencia de transmisión, menor soporte de modos de transmisión descendentes, duración de batería ultra-larga mediante reducción de consumo y operación con cobertura extendida. A los dispositivos de este tipo se les asigno la categoría Cat-M1 (anteriormente conocida como CAT-M). En la Release 13 MTC consigue una reducción adicional del $50 \%$ del coste mediante la restricción del ancho de banda a $1.5 \mathrm{MHz}$, lo cual tiene un fuerte impacto en el diseño del receptor, reduciendo la complejidad del procesamiento de banda base. Esta Release también introduce mejoras de cobertura para ampliar el alcance en $15 \mathrm{~dB}$, permitiendo a los operadores alcanzar dispositivos MTC en condiciones de cobertura pobre como contadores colocados en sótanos. Estas dos mejoras tienen un impacto importante en el diseño de los canales físicos y lógicos que tienen que soportar modos de bajo ancho de banda y cobertura mejorada. Otra mejora clave en Release 13 es la introducción de mejoras en recepción discontinua (eDRX) [6] que está basada en el uso de temporizadores más largos de DRX (recepción discontinua) para alcanzar reducciones adicionales de consumo de potencia. El concepto de DRX 


\begin{tabular}{|c|c|c|c|c|}
\hline & Release-8 & Release-12 & Release-13 & Release-13 \\
\hline & Cat. 1 & Cat. 0 & Cat. M1 & Cat. NB1 \\
\hline Donwlink peak & $10 \mathrm{Mbps}$ & $1 \mathrm{Mbps}$ & $1 \mathrm{Mbps}$ & $200 \mathrm{kbps}$ \\
\hline Uplink peak rate & $5 \mathrm{Mbps}$ & $1 \mathrm{Mbps}$ & $1 \mathrm{Mbps}$ & $144 \mathrm{kbps}$ \\
\hline Number of antennas & 2 & 1 & 1 & 1 \\
\hline Duplex mode & Full duplex & Half duplex & Half duplex & Half duplex \\
\hline UE receive bandwidth & $20 \mathrm{MHz}$ & $20 \mathrm{Mhz}$ & $1.4 \mathrm{MHz}$ & $200 \mathrm{kHz}$ \\
\hline UE transmit power & $23 \mathrm{dBm}$ & $23 \mathrm{dBm}$ & $20 \mathrm{dBm}$ & $23 \mathrm{dBm}$ \\
\hline Complexity & $100 \%$ & $50 \%$ & $20 \%$ & $15 \%$ \\
\hline Use case & $\begin{array}{l}\text { Voice services } \\
\text { for emergency in } \\
\text { elevators,Smart } \\
\text { Grid Management, } \\
\text { Kids/Elderly/Pet tracking }\end{array}$ & $\begin{array}{l}\text { Cat0 is the interim solu- } \\
\text { tion prior Cat-M. Cat } 0 \text { is } \\
\text { used for replacing Cat } 1 \text {, } \\
\text { but cannot replace voice } \\
\text { use cases. }\end{array}$ & $\begin{array}{l}\text { Environment monitoring, } \\
\text { Vehicle tracking }\end{array}$ & $\begin{array}{l}\text { Smart metering, } \\
\text { smart buildings, home } \\
\text { automation }\end{array}$ \\
\hline Availability & Available & Available & 2017 & 2017 \\
\hline
\end{tabular}

Cuadro II

EVOLUTION OF UE CATEGORIES FOR MTC/IOT

consiste en la monitorización de los canales descendentes durante períodos de tiempo limitados. Durante el resto del tiempo el dispositivo se duerme para reducir el consumo de potencia.

En Mayo de 2014, Huawei y Vodafone propusieron un study item para NB-M2M al grupo 3GPP GERAN que rápidamente consiguió un fuerte soporte y creciente atención de otros operadores líderes. En Octubre del mismo año, QC envió una nueva propuesta de narrowband IoT llamada NB-OFDM. En Mayo de 2015, ambas tecnologías se fusionaron en NB-CIoT (NarrowBand Cellular IoT).

Mientras tanto, Ericcson aceleró su investigación en narrowband IoT y propuso NB-LTE (Narrowband LTE) en agosto de 2015. En Septiembre de 2015, el 3GPP aceptó la inclusión de ambas tecnologías como un Work Item en Release 13 [1]. NB-CIoT es un enfoque desde cero (clean slate) promovido por Huawei. La principal diferencia entre NB-LTE y NB-CIoT viene de cuanto de las redes LTE actuales se puede reusar para IoT. NBCIoT requiere nuevos chipsets y no es compatible hacia atrás con ninguna red LTE anterior a Release 13. NB-LTE, por contraste, podría ser completamente integrada en las redes LTE, funcionando en las bandas LTE actuales sin necesidad de una red solapada. En Noviembre de 2015, 3GPP acordó que ambas iniciativas evolucionaran hacia un único estándar llamado Narrowband IoT (NB-IoT). NBIoT especifica un nuevo acceso radio para IoT celular, basado en buena medida en una variante no compatible hacia atrás de LTE, que se orienta a una cobertura mejorada en interiores, soporte para un número masivo de dispositivos de baja tasa de transmisión, escenarios poco sensibles al retardo, dispositivos de ultra bajo coste, bajo consumo de potencia y una arquitectura de red optimizada. Así, NB-IoT se ha convertido en la principal solución de 3GPP para redes de área extensa y bajo consumo LPWAN (Low Power Wide Area Network), reemplazando a las propuestas NB-LTE y NB-CIoT (Cellular IoT).

En Junio de 2016 3GPP completó la estandarización de NB-IoT, la nueva tecnología radio de banda estrecha desarrollada para la Internet de las cosas (IoT). Los UEs NB-IoT se denominan como Cat-NB1 (también referen- ciados en la literatura como Cat-M2). La reducción de su complejidad, comparada con Cat.1 es de hasta el $90 \%$. Habiendo terminado la estandarización de NB-IoT en Junio de 2016, llevó solamente 9 meses estandarizar esta nueva tecnología después de la fase de estudio, lo que demuestra la importancia de IoT para el 3GPP.

El trabajo llevado a cabo en el grupo de GSM/EDGE dentro de 3GPP para cobertura extendida de GSM para IoT (EC-GSM-IoT), se ha integrado en estos dos proyectos: eMTC (LTE-M) y NB-IoT.

En paralelo con estos procesos de estandarización llevados a cabo en 3GPP, hay otras iniciativas que operan de forma no licenciada. Este es el caso de LoRaWAN, Sigfox y OnRamp Wireless, Wihtless -N \& -P, etc. La mayoría de estas redes hace uso de las bandas de frecuencia no licencias ISM para uso industrial, científico y médico. Testas tecnologías están actualmente disponibles, han sido desplegadas y cumplen con los cuatro factores para LPWAN (largo alcance, muy baja potencia, baja tasa de transmisión y bajo coste). Algunas están basadas en protocolos apoyados por alianzas industriales como la LoRaWAN Alliance y Wightless SIG, otros están basados en protocolos propietarios y otros son estándares en progreso. Además de las diferencias tecnológicas, hay que destacar que algunas de estas tecnologías están sujetas a nuevos modelos de negocio. En un plano económico, una ventaja adicional de NB-IoT respecto de estas tecnologías, puede venir del mayor consenso entre operadores, la integración en las redes celulares y de la resultante economía de escala.

\section{RED DE ACCESO RADIO NB-IOT}

NB-IoT proporciona acceso radio a servicios de red usando una capa física [8] optimizada para muy bajo consumo y coste. El ancho de banda del canal completo es de $180 \mathrm{kHz}$, la separación entre subportadoras puede ser de $15 \mathrm{kHz}$ o de $3.75 \mathrm{kHz}$ (solamente en el uplink), el esquema de modulación más alto es QPSK, hay soporte para operación FDD y half-duplex. El enlace descendente de NB-IoT se basa en OFDM y el esquema de transmisión usa un único bloque de recursos físicos (PRB). El enlace 
ascendente se basa en single-carrier FDMA. Para la transmisión ascendente, hay dos modos de operación posibles, transmisión de tono único (single tone) y multi-tono (multi-tone). En tono único, está permitido tanto $3.75 \mathrm{~Hz}$ como $15 \mathrm{KHz}$ de espaciado entre subportadoras, mientras que en multi-tono solamente se puede usar espaciado de $15 \mathrm{KHz}$. La transmisión multi-tono permite agrupar conjuntos de 3,6 o 12 subportadoras. De forma adicional, la duración mínima de las unidades de recursos usadas en la planificación depende del número de subportadoras asignadas y del modo de operación, yendo desde $1 \mathrm{~ms}$ en transmisión multi-tono con 12 subportadoras, a $32 \mathrm{~ms}$ en transmisión de tono único de $3.75 \mathrm{kHz}$.

Hay ciertas funciones de protocolos E-UTRA (Evolved Universal Terrestrial Radio Access) que soportan todos los UEs Rel-8 que sin embargo no son usadas por los UEs NB-IoT: movilidad entre distintas tecnologías radio (inter-RAT), traspasos, reporte de medidas, funciones de alerta públicas, tasas de datos garantizadas (GBR), grupos cerrados de usuarios (CSG), soporte de femtoceldas (HeNBs), de nodos intermedios (relaying), agregación de portadoras, conectividad dual, NAICS (Network Assisted Interference Cancellation and Suppression ), comunicaciones de difusión y multipunto MBMS, servicios de tiempo real, evitación de interferencia para coexistencia interna, interoperabilidad con WLAN asistida por la red, comunicación y descubrimiento entre dispositivos (sidelink), reducción del número de drive tests (MDT), llamadas de emergencia y conmutación a llamadas de circuitos (CS fallback).

En NB-IoT, se puede soportar el posicionamiento usando la arquitectura existente de Servicios de Localización (LCS) mediante medidas únicamente en el eNB. Se introducen cinco nuevos canales:

- Narrowband Physical Broadcast

- Narrowband Physical Downlink Shared Channel (NPDSCH). Transporta el DLSCH (Downlink Shared Channel) y el PCH (Paging Channel) para UEs NBIoT.

- Narrowband Physical Downlink Control Channel (NPDCCH). Informa al UE NB-IoT de la asignación de recursos del $\mathrm{PCH}$ y del DL-SCH, y transporta el 'scheduling grant' asignado al UE NB-IoT para transmisión en el enlace ascendente.

- Narrowband Physical Uplink Shared Channel (NPUSCH). Transporta el UL-SCH (Uplink Shared Channel) y la confirmación ACK/NACKs Hybrid ARQ en respuesta a la transmisión descendente hacia el dispositivo UE NB-IoT.

- Narrowband Physical Random Access Channel (NPRACH). Transporta el preámbulo de acceso aleatorio enviado por el UE NB-IoT.

Respecto de la capa de control de acceso al medio (MAC), NB-IoT introduce cambios para reducir el consumo de potencia y para hacer el funcionamiento del planificador más flexible y simple. Debido a los menores requisitos de tasa de transmisión, se utiliza un único proceso HARQ. Esto permite eliminar el identificador de
HARQ de las asignaciones del planificador y así usar un menor número de bis para una mayor eficiencia y robustez. Adicionalmente, las retransmisiones en el enlace ascendente (UL) dejan de ser síncronas sino siempre adaptativas y asíncronas tanto en UL como en DL. Esto proporciona a la red un control más ajustado de planificación del enlace ascendente, mientras que protege de retransmisiones periódicas indeseadas ya que ahora se generan únicamente cuando sean solicitadas explícitamente.

Otras mejoras en las capas 2 y 3 de NB-IoT incluyen la reducción de los tamaños máximos de los elementos de datos (SDU) y control (PDU) de la capa de convergencia de paquetes de datos (PDCP). De los anteriores 8188 octetos se pasa a un máximo de 1600 bytes. Este es un compromiso razonable ya que el tráfico tradicional de internet no excede de 1500 bytes por paquete IP.

Mientras que un dispositivo NB-IoT está en modo conectado, es posible configurar recepción discontinua (DRX) con ciclos de hasta 10.24 segundos. Respecto de la configuración de Paging, cuando se habilita DRX en modo de espera (idle), en NB-IoT la máxima duración del ciclo de DRX es de 10485.76 segundos (2.91 horas). Esto permite ahorros de energía muy notables cuando el caso de uso es compatible con la alta latencia que tendrá asociada el establecimiento de conexión.

\section{ARQUITECTURA NB-IOT}

La arquitectura de NB-IoT simplifica la arquitectura existente del núcleo de red (EPC) con el propósito de cumplir con los requisitos y modelos de tráfico identificados en [1] y mostrados en la Tabla III. La transmisión de pequeños datos se basa en una arquitectura simplificada que está orientada a transportar estos pequeños bloques de información sobre mensajes de señalización con el número de red o Non Access Stratum (NAS). Se usa un nodo dedicado del núcleo de red para un perfil CIoT (terminología 3GPP para Internet de las cosas), proporcionando funciones combinadas de plano de control y de usuario, e.g. agregando funciones que tradicionalmente residían en el MME (Mobility Management Entity) y en el SGW (Serving Gateway), y en algunas instancias de PGW (Packet Data network Gateway) en una nueva entidad lógica llamada C-SGN( CIoT Serving Gateway Node). C-SGN puede implementarse para soportar únicamente la funcionalidad necesaria para casos de uso CIoT. S1lite es una versión optimizada de S1-C ( el plano de control del interfaz S1 que se basa en el protocolo S1AP) entre el eNodeB y el MME. Del S1-C solamente se soportan los mensajes S1AP necesarios (y dentro de los mensajes solamente aquellas partes requeridas) para los procedimientos CIoT. Se soportan también procedimientos de seguridad optimizados así como algunas funcionalidades para permitir el transporte de datos. El plano de usuario se transporta en estos mensajes S1AP modificados para permitir un manejo eficiente de datos pequeños. Los túneles del plano de datos del interfaz S1-U (definido en TS 23.401[9]) no son necesarios.

Esta arquitectura permite que ciertas modificaciones (por ejemplo, soporte nativo de SMS en el dominio de 


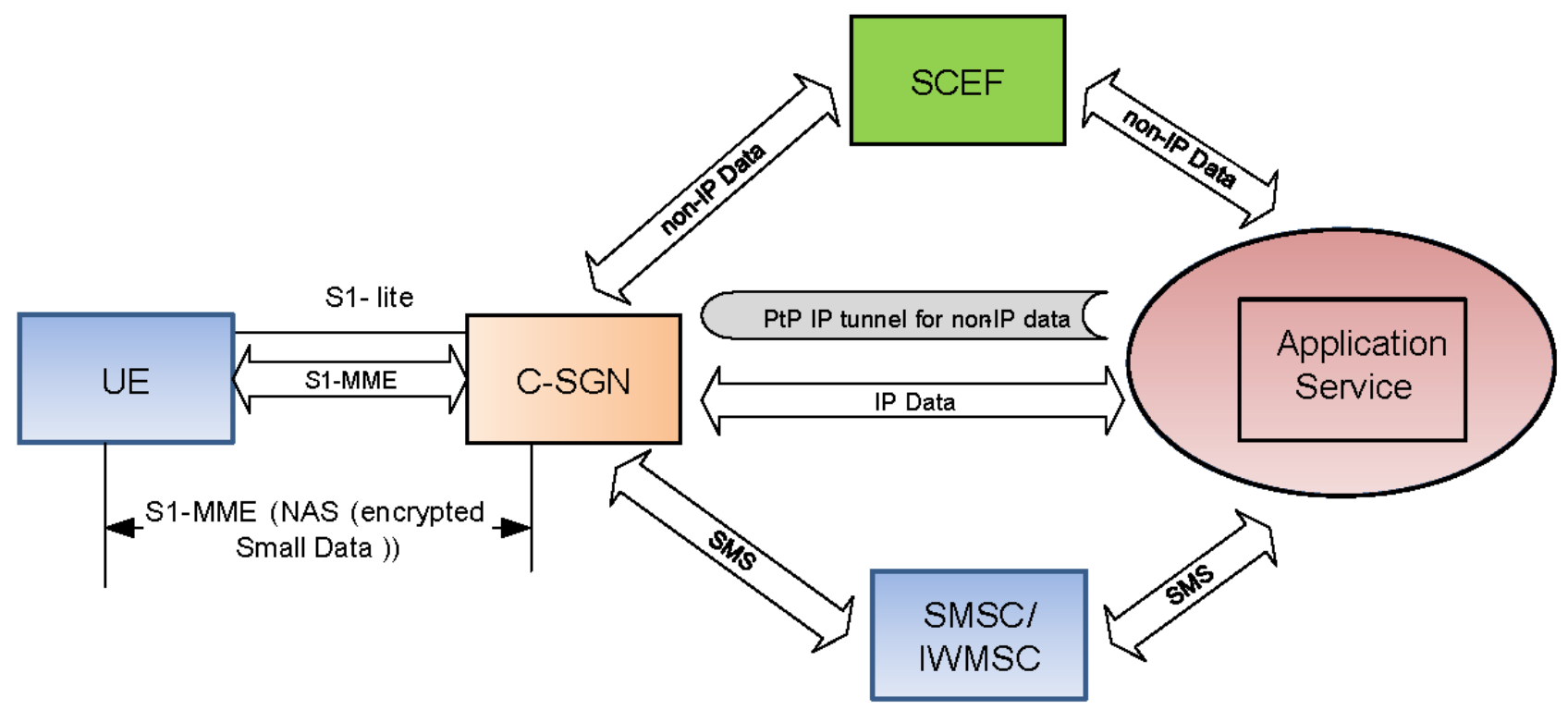

Figura 1. LTE Architecture for NB-IoT

\begin{tabular}{|c|c|c|c|c|}
\hline Category & Application example & UL Data Size & DL Data Size & Frequency \\
\hline $\begin{array}{l}\text { Mobile Autonomous Re- } \\
\text { porting (MAR) exception } \\
\text { reports }\end{array}$ & $\begin{array}{l}\text { smoke alarm detectors, } \\
\text { power failure notifications } \\
\text { from smart meters, tamper } \\
\text { notifications etc. }\end{array}$ & 20 bytes & $\begin{array}{l}0 \text { ACK payload size is as- } \\
\text { sumed to be } 0 \text { bytes }\end{array}$ & $\begin{array}{l}\text { Every few months; Every } \\
\text { year }\end{array}$ \\
\hline $\begin{array}{l}\text { Mobile Autonomous Re- } \\
\text { porting (MAR) periodic } \\
\text { reports }\end{array}$ & $\begin{array}{l}\text { smart utility } \\
\text { (gas/water/electric) } \\
\text { metering reports, } \\
\text { smart agriculture, smart } \\
\text { environment etc. }\end{array}$ & $\begin{array}{l}20 \text { bytes with a cut off } \\
\text { of } 200 \text { bytes i.e. payloads } \\
\text { higher than } 200 \text { bytes are } \\
\text { assumed to be } 200 \text { bytes. }\end{array}$ & $\begin{array}{l}50 \% \text { of UL data size ACK } \\
\text { payload size is assumed to } \\
\text { be } 0 \text { bytes }\end{array}$ & $\begin{array}{l}1 \text { day }(40 \%), 2 \text { hours } \\
(40 \%), 1 \text { hour }(15 \%) \text {, and } \\
30 \text { minutes }(5 \%)\end{array}$ \\
\hline Network Command & $\begin{array}{l}\text { Switch on/off, device trig- } \\
\text { ger to send uplink report, } \\
\text { request for meter reading }\end{array}$ & $\begin{array}{l}0-20 \text { bytes } 50 \% \text { of cases } \\
\text { require UL response. }\end{array}$ & 20 bytes & $\begin{array}{l}1 \text { day }(40 \%), 2 \text { hours } \\
(40 \%), 1 \text { hour }(15 \%) \text {, and } \\
30 \text { minutes }(5 \%)\end{array}$ \\
\hline $\begin{array}{l}\text { Software } \\
\text { te/reconfiguration model }\end{array}$ & Software patches/updates & $\begin{array}{l}200 \text { bytes with a cut off } \\
\text { of } 2000 \text { bytes i.e. payload } \\
\text { higher than } 2000 \text { bytes are } \\
\text { assumed to be } 2000 \text { bytes. }\end{array}$ & $\begin{array}{l}200 \text { bytes with a cut off } \\
\text { of } 2000 \text { bytes i.e. payload } \\
\text { higher than } 2000 \text { bytes are } \\
\text { assumed to be } 2000 \text { bytes. }\end{array}$ & 180 days \\
\hline
\end{tabular}

Cuadro III

Traffic Models for Cellular IoT [3GPP TR 45.820[1]]

paquetes, registro sin conexión PDN, NAS simplificado, etc.) se apliquen solamente a UEs CIoT sin requerir que los procedimientos mantengan compatibilidad hacia atrás con otros UEs.

En NB-IoT, para optimizar la señalización, se han introducido dos soluciones además del tradicional establecimiento de conexión RRC (Radio Resource Control). La primera solución, Data-over-NAS (DONAS), es obligatoria y es una optimización del plano de control basada en la solución 2 propuesta en [7]. DONAS permite la transmisión de datos sin tener que activar el plano de usuario. Implementando solamente esta opción permite implementaciones de UE con menos requisitos relativos al plano de usuarios, como el soporte para podo sin confirmación (UM) en RLC, múltiples canales lógicos dedicados, DTCH, re-establecimiento RLC ... La segunda solución es opcional y está basada en la solución 1 (especificada en [7]). La suspensión y restauración de RRC introduce mejoras para deshabilitar y recuperar el plano de usuarios de forma eficiente. En el plano de control optimizado, los datos se envían sobre Non Access Stratum (NAS), directamente desde la Mobility Management Entity (MME) en el núcleo de red hacia el UE sin interacción de la estación base.

Los procedimientos de suspensión y recuperación RRC reducen la sobrecarga de señalización y también mejoran la duración de la batería del UE. La arquitectura de protocolos de NB-IoT y LTE se separa en plano de usuario y control. El plano de control consiste en protocolos que gestionan las portadoras de acceso radio y la conexión entre el UE y la red. La capa más alta del plano de control se denomina capa de no acceso o Non-Access Stratum (NAS) y realiza el intercambio de señalización entre el UE y el núcleo de red (EPC), pasando de forma transparente a través de la red radio. Es el responsable de la autenticación, control de seguridad, gestión de movilidad y gestión de portadoras. La capa de acceso o Access Stratum (AS), es el nivel funcional por debajo de 
NAS, y en el plano de control consiste en el protocolo de control de recursos radio (RRC). RRC configura los planos radio de control y de usuario de acuerdo al estado de la red. Hay dos estados principales a nivel RRC, inactivo (RRC_Idle) o conectado (RRC_Connected), y la entidad RRC controla la conmutación entre estos estados. En estado RRC_Idle, la red sabe que el UE está presente en la red y que que se puede ser alcanzado en caso de una llamada o conexión entrante. En estado RRC_Connected el UE tiene una conexión radio activa con el eNodeB, la red sabe la posición del UE a nivel de celda y el UE puede transmitir y recibir datos. Cuando no hay tráfico se libera la conexión, pasando a RRC_Idle, para ahorrar batería y recursos radio. Los procedimientos de suspensión y recuperación reducen la sobrecarga requerida para la transición del estado del UE de Idle a Connected para establecer el plano de usuario, y de vuelta a estado Idle, reduciendo los mensajes de señalización requeridos en comparación con la operativa tradicional.

De cara a soportar los procedimientos de suspensión/recuperación RRC, MME, eNodeB y UE deben incluir nuevas funcionalidades. El MME, que es responsable de gestionar la movilidad del UE, debe soportar nuevos procedimientos S1AP, en particular el procedimiento disparado por los procedimientos 'RRC Suspend' y 'RRC Resume' en el interfaz radio. El MME tiene asimismo que almacenar la información de Contexto del UE cuando la conexión RRC es suspendida y la asociación S1AP se mantiene. El eNodeB debe permitir el almacenamiento del contexto del UE así como de los parámetros relacionados con la asociación S1AP cuando el UE está en el nuevo estado CIoT RRC-Idle. Además, el eNodeB tiene que soportar los nuevos procedimientos para suspender y recuperar la conexión. Estos procedimientos deberán implementarse entre el eNB y el UE, así como entre el eNB y el MME. Finalmente el UE debe almacenar la información relevante de AS cuando el UE entra en estado CIoT RRC_Idle y soporta los procedimientos de suspensión y recuperación de la conexión RRC.

Data-overNAS se considera como la base del trabajo normativo para soportar transmisiones infrecuentes de datos (para datos IP, no-IP y SMS). El soporte de DONAS es obligatorio tanto para el UE como para la red. Esta solución permite un soporte eficiente para pequeñas transmisiones infrecuentes para IoT y soporte para datos no-IP. La solución se basa en una arquitectura ligera del núcleo de red mostrada en la Figura 1. Esta solución encapsula pequeñas cantidades de datos en el enlace ascendente en el mensaje inicial NAS, que se ha extendido, y usa un mensaje NAS descendente adicional para transportar respuestas pequeñas. De esta forma, se puede evitar el esfuerzo requerido para establecer el plano de usuario, e.g. mensajes RRC asociados y establecimiento de seguridad AS. No hay establecimiento de DRBs y portadoras S1-U. Los datos se pueden entregar también a través del SCEF (Service Capability Exposer Function), que se usa para la entrega de datos no-IP sobre el plano de control. El elemento SCEF es parte de las mejoras de arquitectura

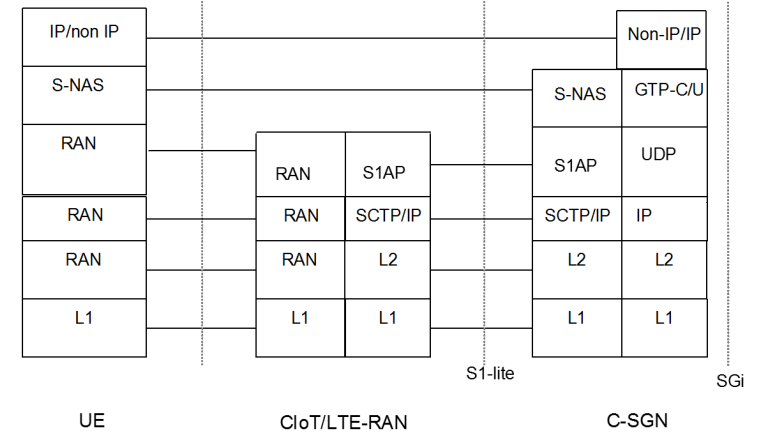

Figura 2. Protocol stack for NB-IoT

exposición de capacidades de servicios (AESE) [10] introducidas en Release 13, que muestra los servicios de red a terceras partes. La implementación de esta solución también implica modificaciones en los procedimientos disponibles en el UE, eNodeB y C-SGN/MME para soportar e.g. la gestión de sesiones 'NAS small data' y el registro sin activación de portadoras.

La pila de protocolos resultante tras la adopción de estas dos soluciones es la pila simplificada mostrada en la Figura 2, donde S-NAS indica una señalización NAS simplificada.

\section{UNA PERSPECTIVA DE PRUEBA Y MEDIDA}

Como se ha explicado en detalle, NB-IoT es una tecnología completamente nueva que busca adaptar las redes celulares a las demandas de conectividad y a la problemática de los dispositivos de bajo coste como sensores y contadores. Proporcionando conectividad directa, sin intermediarios, a todos los dispositivos, se abre un amplio abanico de nuevos escenarios y de nuevas oportunidades para casos de uso, servicios y dispositivos que aparecerán en un futuro y que no se hayan imaginado a día de hoy.

Esta revolución tecnológica no solamente va a traer grandes ventajas, sino que va a presentar importantes retos y desafíos a muy distintos niveles a los actores involucrados. Desde el punto de vista de un fabricantes de nuevos dispositivos o de un operador que tiene que incorporarlos a su red surge la necesidad de verificar los diseños de radiofrecuencia, comprobar el procesamiento de banda base, asegurar una correcta interoperabilidad en los distintos protocolos, verificar la estabilidad de los dispositivos y la medir las dinámicas de consumo de potencia y longevidad de las baterías. Para investigadores que buscan proponer entender la tecnología y proponer evoluciones futuras, es importante entender las dependencias entre los distintos parámetros y poder ajustar sus modelos caracterizando el impacto de la configuración de red en el enlace de comunicación NB-IoT y en los servicios que hacen uso del mismo. Para todo ello, proponemos el uso de un elemento de referencia como el UXM E7515A de Keysight Technologies [11], que ha sido validado para NB-IoT y eMTC CAT-M por el Global Certification Forum (GCF).

Usando como ejemplo un objetivo clave y ambicioso de NB-IoT, como es conseguir verificar que la duración 


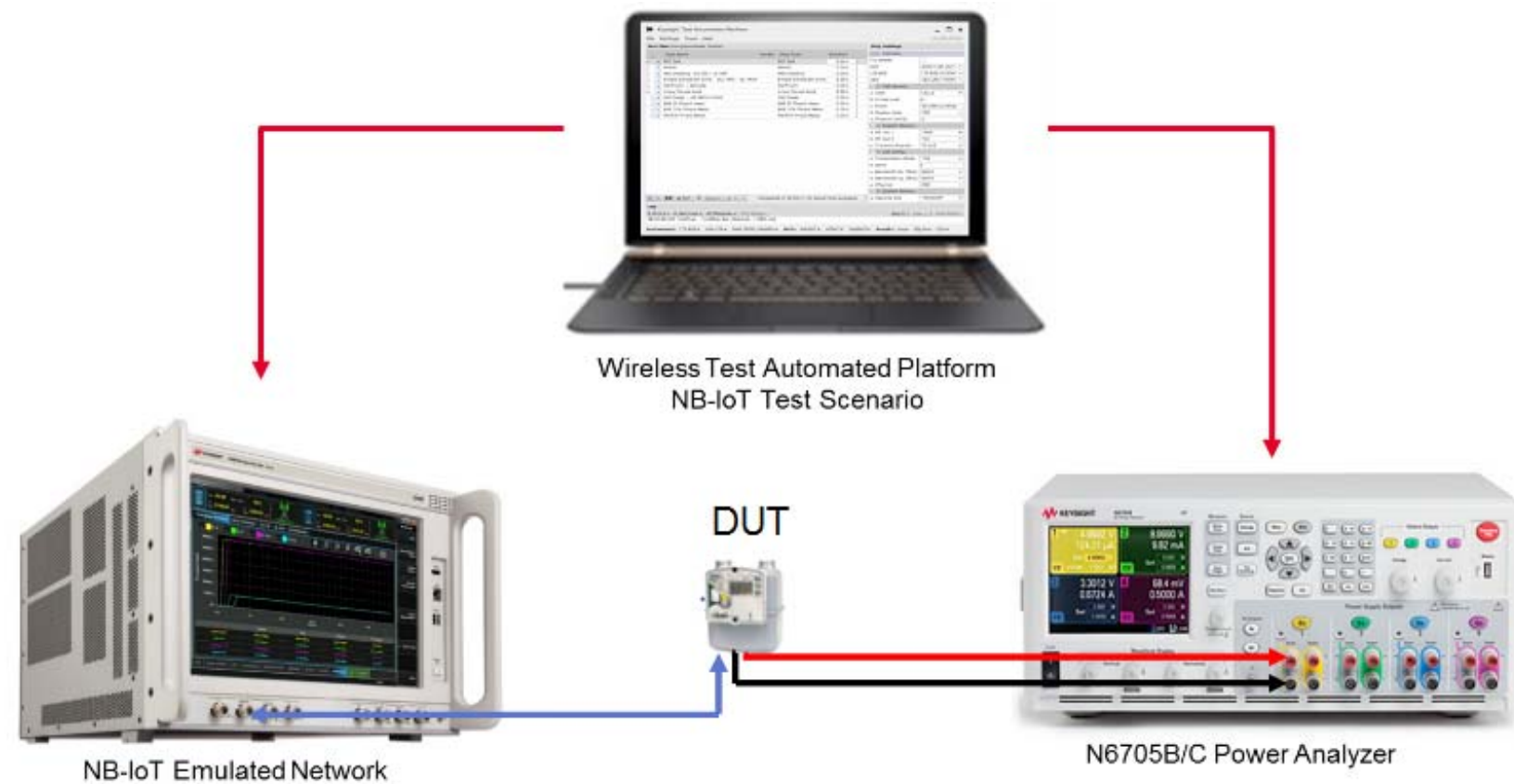

Figura 3. Entorno de pruebas y medidas para NB-IoT

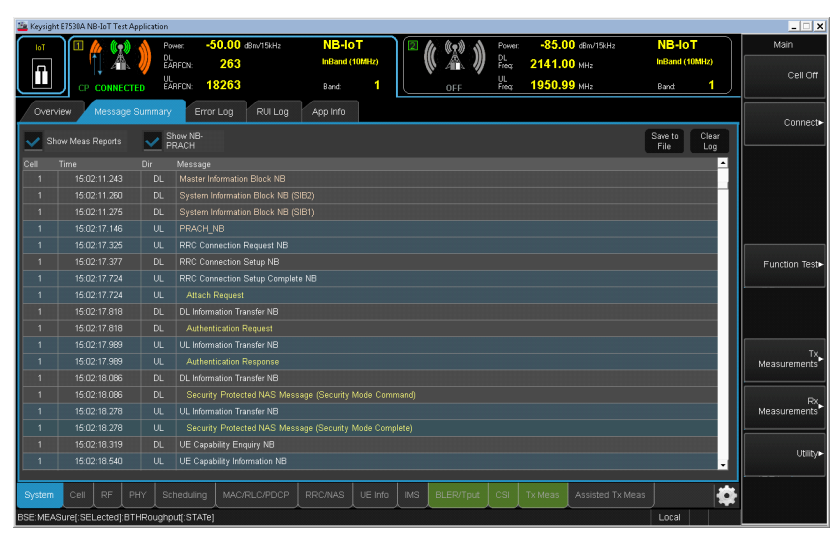

Figura 4. Mensajes de control entre el UE y el eNodeB

de batería alcance 10 años, se requiere de herramientas de análisis de consumo de energía muy precisas y de mecanismos para realizar experimentos en condiciones realistas de funcionamiento, ya que el consumo real de un dispositivo variará en buena medida en función de las condiciones en las que se encuentre. Como se muestra en la Figura 3, esto es posible combinando las capacidades de emulación de red del UXM con un analizador de potencia de precisión como el N6705B/C de Keysight.

Conectando un dispositivo NB-IoT real al UXM, es posible reproducir una red con múltiples celdas NB-IoT, y controlar y monitorizar tanto la señalización (ver Figura 4) y los parámetros de configuración los protocolos de bajo nivel como la potencia recibida por el dispositivo y las condiciones de propagación incluyendo canales con desvanecimientos e interferentes, como se muestra en la Figura 5. Por otra parte, utilizando la aplicación de medida de la serie $\mathrm{X}$ integrada en el UXM, es posible realizar

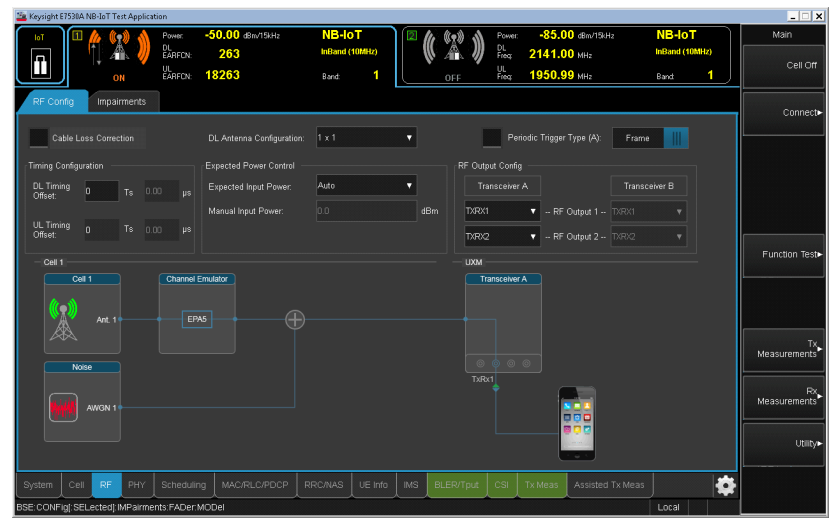

Figura 5. Emulación de propagación radio y de interferentes

numerosas medidas de RF y de banda base. En la Figura 6 se muestra un ejemplo de análisis de modulación de la señal NPUSCH transmitida por un dispositivo NB-IoT. Además de la constelación IQ, es posible observar el espectro de potencia y consultar multiples indicadores de calidad, así como verificar la potencia transmitida.

En pruebas de concepto iniciales, hemos constatado el tremendo impacto que pueden llegar a tener las condiciones de propagación y la configuración de red, aumentando el tiempo de ida y vuelta de la comunicación en órdenes de magnitud en función del número de repeticiones necesarias. En la Figura 7 se muestra un escenario de conectividad IP extremo a extremo en condiciones favorables, aún así el tiempo de ida y vuelta es de unos 200 ms como se puede apreciar por la separación entre el tráfico DL y UL.

Asimismo, debido la capacidad extremadamente reducida de NB-IoT, en pruebas iniciales hemos verificado que 


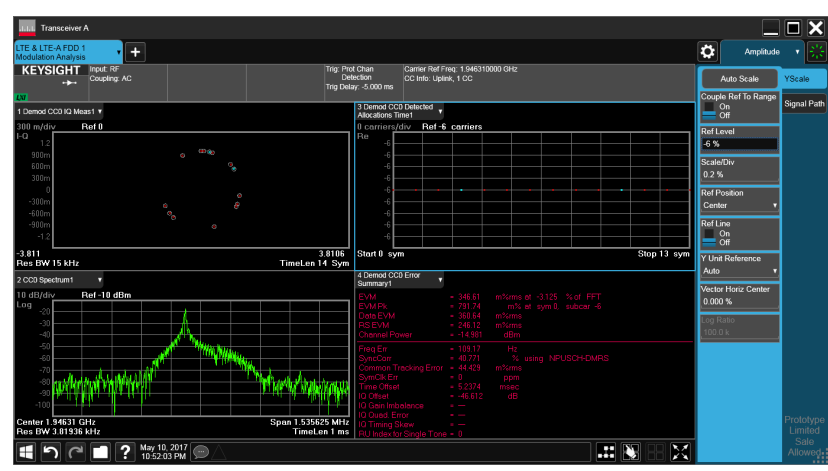

Figura 6. Medidas de espectro, potencia de señal y calidad

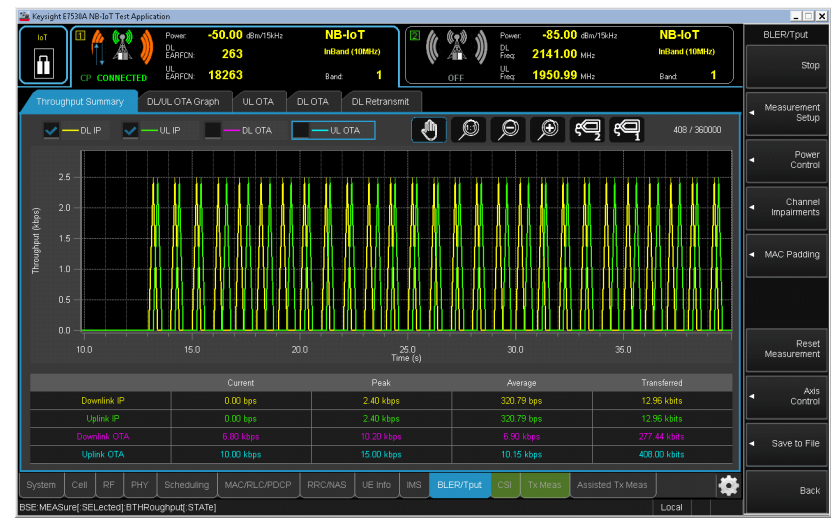

Figura 7. Tráfico IP extremo a extremo de baja tasa binaria

puede ser muy dañino que cualquier tráfico aunque sea residual proveniente de un equipo conectado a un dispositivo NB-IoT. Por tanto, en donde los dispositivos NB-IoT vayan a funcionar como modem, hemos identificado la necesidad de utilizar de forma sistemática firewalls u otros mecanismos que dejen pasar solamente el tráfico deseado. Otra interesante lección aprendida es que algunas de las herramientas tradicionales de prueba de tráfico tienen problemas para operar en escenarios de muy baja tasa de transmisión y particularmente ante picos de retardo muy elevados como los presentes en algunas configuraciones de NB-IoT, por ello es recomendable utilizar soluciones contrastadas.

En trabajos futuros analizaremos de forma detallada las dependencias entre los principales parámetros, realizando experimentos repetibles y automáticos mediante el software de control Wireless Test Automation Platform.

\section{CONCLUSIONES}

Mientras que eMTC (LTE-M) es una tecnología puramente LTE, NB-IoT se basa en un nuevo interfaz radio que puede coexistir con los sistemas LTE, UMTS y GSM actuales. Respecto de los casos de uso de ambas tecnologías, la principal diferencia es la movilidad y la tasa de transmisión. NB-IoT ofrece tasas y movilidad reducidas en comparación con eMTC, pero permite una mayor cobertura y densidad, lo que la hacen más adaptada a escenarios IoT, como las redes de sensores mientras que eMTC presentaría ventajas en nichos como el de los dispositivos wearables. En Releases posteriores, se tiende a introducir mejoras adicionales para IoT en la forma de soporte para multicast, reducción de latencia para posibilitar escenarios (V2V), posicionamiento, movilidad y mejoras en la continuidad de servicio y nuevas clases de potencia entre otras.

\section{AGRADECIMIENTOS}

Este trabajo ha sido financiado por el Ministerio Español de Economía y Competitividad (TIN2015-67083R), FEDER y el programa de investigación e innovación Horizonte 2020 de la Unión Europea (grant agreement No 688719).

\section{REFERENCIAS}

[1] 3GPP TR 45.820 Cellular system support for ultra-low complexity and low throughput Internet of Things (CIOT)

[2] 3GPP TS 36.101 Evolved Universal Terrestrial Radio Access (EUTRA); User Equipment (UE) radio transmission and reception

[3] 3GPP 36.888, Study on provision of low-cost Machine-Type Communications (MTC) UEs based on LTE

[4] J. Gozalvez, "New 3GPP Standard for IoT [Mobile Radio],ïn IEEE Vehicular Technology Magazine, vol. 11, no. 1, pp. 14-20, March 2016.

[5] S. Landström, J. Bergström, E. Westerberg, D. Hammarwall, "NBIoT: A sustainable technology for connecting billions of devices", in Ericsson Technology Review, vol. 93, no. 3, pp. 2-11, April 2016

[6] 3GPP TR 23.770, Study on System Impacts of Extended DRX Cycle for Power Consumption Optimization

[7] 3GPP TR 23.720 Study on architecture enhancements for Cellular Internet of Things

[8] 3GPP TS 36.300 LTE; Evolved Universal Terrestrial Radio Access (E-UTRA) and Evolved Universal Terrestrial Radico Access Network (E-UTRAN); Overall description; Stage 2

[9] 3GPP TS 23.401 General Packet Radio Service (GPRS) enhancements for Evolved Universal Terrestrial Radio Access Network (E-UTRAN) access

[10] 3GPP TR 23.708 Architecture Enhancements for Service Capability Exposure (AESE)

[11] UXM Wireless Test Set, http://www.keysight.com/en/ pd-2372474-pn-E7515A/uxm-wireless-test-set?cc=US\&lc=eng 